\title{
Research Paper: Investigating the Effects of Different Working Postures on Cognitive Performance
}

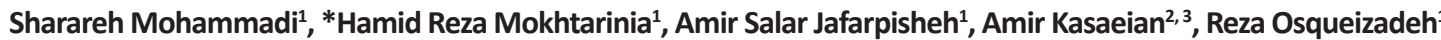

1. Department of Ergonomics, University of Social Welfare and Rehabilitation Sciences, Tehran, Iran.

2. Hematology-Oncology and Stem Cell Research Center, Tehran University of Medical Sciences, Tehran, Iran.

3. Hematologic Malignancies Research Center, Tehran University of Medical Sciences, Tehran, Iran.

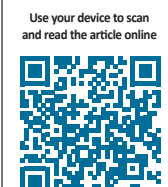

Clttation: Mohammadi Sh, Mokhtarinia HR, Jafarpisheh AS, Kasaeian A, Osqueizadeh R. [Investigating the Effects of Different Working Postures on Cognitive Performance (Persian)]. Archives of Rehabilitation. 2018; 18(4):268-277. https://doi. org/10.21859/JREHAB.18.4.1

\section{https://doi.org/10.21859/JREHAB.18.4.1}

Received: 17 Aug. 2017 Accepted: 05 Nov. 2017

Keywords:

Posture, Static work, Reaction time, Selective attention, Cognitive performance

\section{ABSTRACT}

Objective Individuals performance at the workplace is affected by different adopted postures. Sitting postures are mostly used during office work. Improper sitting postures may cause muscle fatigue and discomfort in the spine and also result in mental workload. Poor posture may result in human errors and delay in information processing. Previous studies have demonstrated the relationship between static sitting postures and cognitive factors such as reaction time to an auditory stimulus. Also, some recent studies have demonstrated the effect of static postures on cognitive performance. To our knowledge, none of the studies had considered the sitting and standing postures effects on cognitive performance simultaneously. The aim of this study was to evaluate cognitive performance during three different static working postures: standard sitting, standing, and self-selected sitting postures.

Materials \& Methods This semi-experimental design study was conducted on the twenty-nine healthy students (aged 20-30 years). The non-probability sampling method was selected. All participants provided their written informed consent. Participants with no musculoskeletal disorders, heart disease, spine surgery, and history of depression and stress were selected. Their depression status and level of stress were measured using Beck questionnaire. The study was approved by the Ethics Committee of University of Social Welfare and Rehabilitation Sciences. Subjects performed complex Stroop test and typing activity in an office-like laboratory setting at the University of Social Welfare and Rehabilitation Sciences. Stroop test lasted about 9-12 minutes, and a typing task was also designed for approximately 5 minutes for all subjects. Three random postures were selected based on common postures used in the office. The effects of the postures (standard standing, standard sitting, and self-selected sitting posture) on dependent variables such as total test duration and reaction time to congruent and incongruent stimulus, number of typing words and number of typing error were assessed with Repeated Measures ANOVA. To examine the differences between groups, the paired t-test was used.

Results The results demonstrated that reaction time measure and number of typing error (accuracy) were affected by postures $(\mathrm{P}=0.001)$. Post hoc analysis demonstrated that reaction time was significantly different between self-selected sitting posture and standard sitting posture $(P=0.001)$, self-selected sitting posture and standard standing posture $(P=0.043)$, standard sitting posture and standard standing posture $(P=0.001)$. With considering the average amount of reaction time to congruent and incongruent cases, it was observed that there is less reaction time in the standardized sitting posture $(\mathrm{M}=628.67 \mathrm{~ms})$ compared to the self-selected sitting posture $(M=689.41 \mathrm{~ms})$ and standard standing posture $(M=675.16 \mathrm{~ms})$. Also, among the three postures studied a significant difference was observed in the number of typing error words $(P=0.001)$. The number of typing error (accuracy) was lower in this posture compared to the two other postures $(\mathrm{M}=1.58)$.

Conclusion This study demonstrates that cognitive performance is affected by working postures. This study demonstrates that standard sitting posture is the best posture. Therefore, it is recommended that sitting posture can help in increasing cognitive performance in the workplace.

\section{* Corresponding Author:}

Hamid Reza Mokhtarinia, PhD

Address: Department of Ergonomics, University of Social Welfare and Rehabilitation Sciences, Tehran, Iran.

Tel: +98 (912) 2495248

E-Mail: hrmokhtarinia@yahoo.com 


\title{
بررسى تأثير يوسجر هاى مختلف كارى بر عملكرد شناختى دانشجويان
}

\author{
شراره محمدى'، "حميدرضا مختارىنيا'، اميرسالار جعفرييشه'، امير كسائيان “، رضا اسكوئىزاده' \\ 1- ا-كروه اركونومى، دانشكاه علوم بهزيستى وتوانبخشى، تهران، ايران.

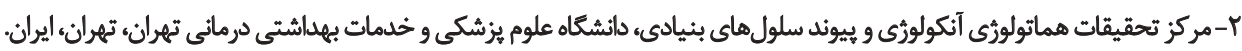

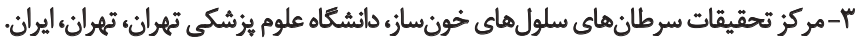

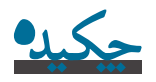

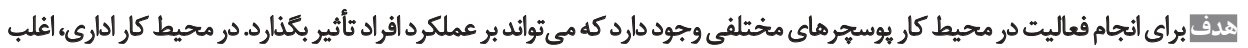

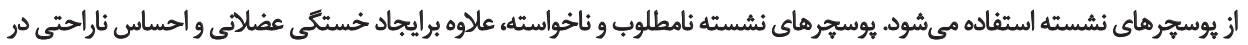

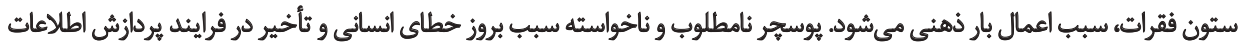

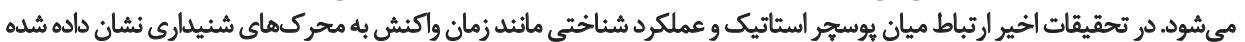

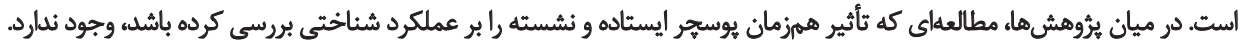

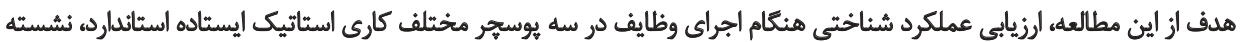

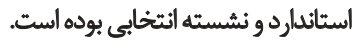

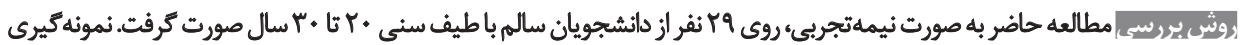

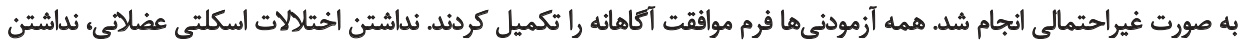

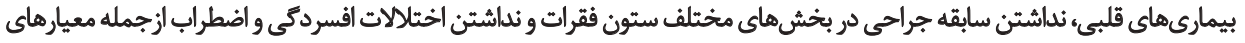

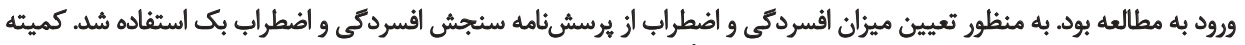

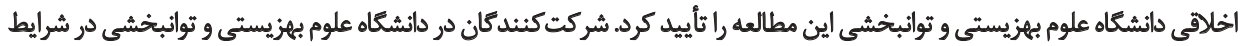

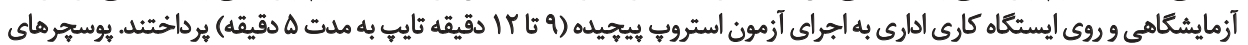

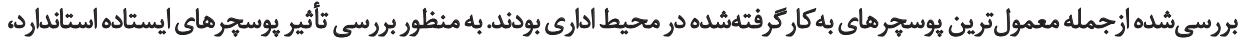

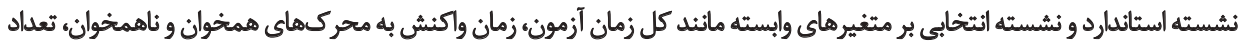

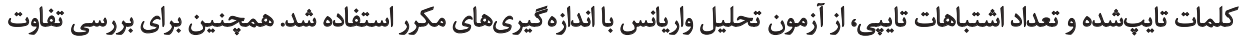

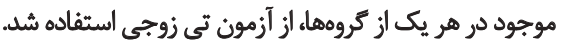

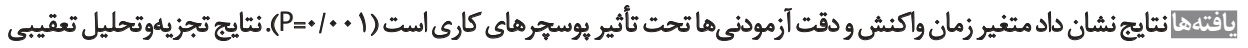

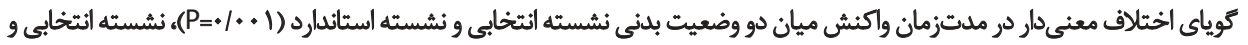

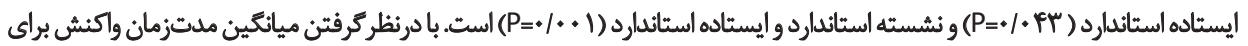

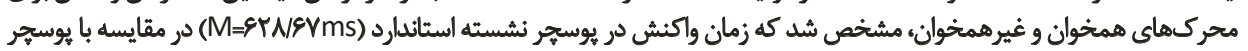

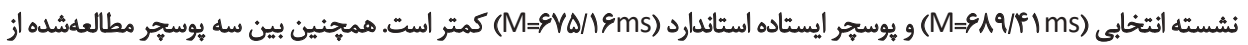

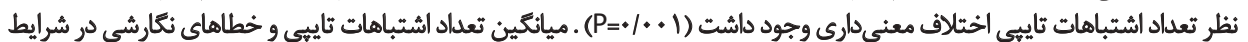

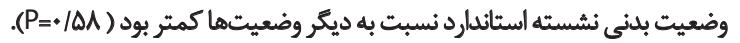

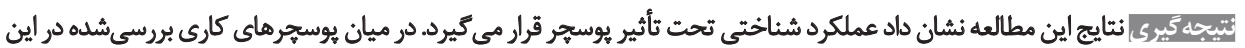

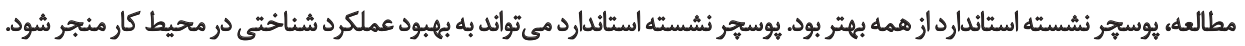

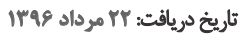

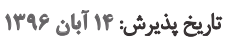


نشسته و ايستاده و برخى از عملكردهاي شناختى مانيند زمان

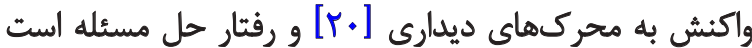

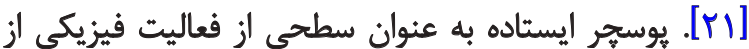

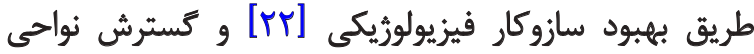

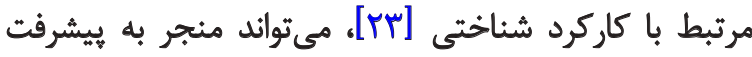

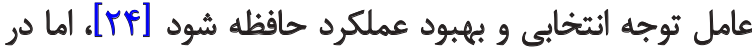

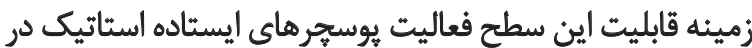

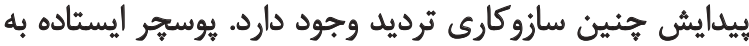

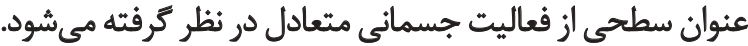

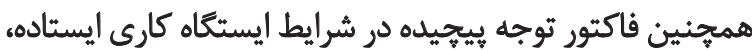

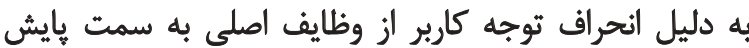
موقعيت خود كاهش مي يابد [rهاف] انقباض عروق، اختلال در خونرسانى و احساس خستكى، ازجمله

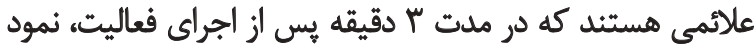

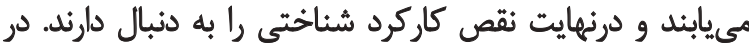

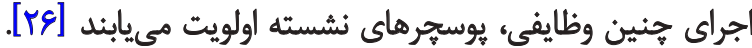

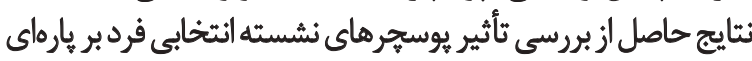

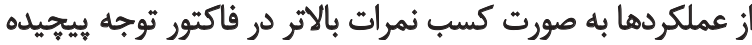

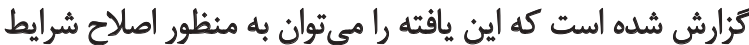

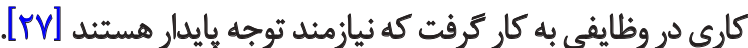

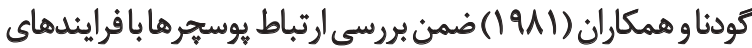

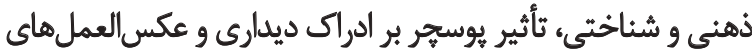

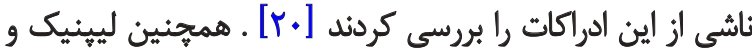

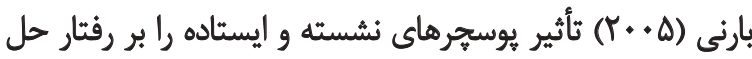

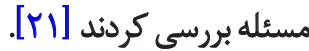
طى سالهاى اخير، مداخلات با هدف كاهش مدتزمان

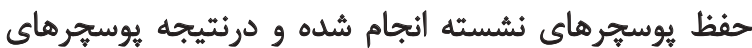

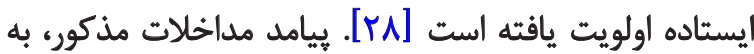

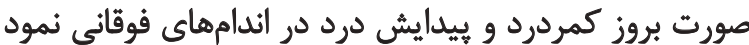

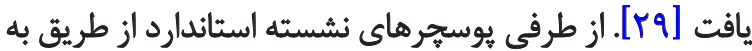

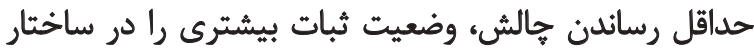

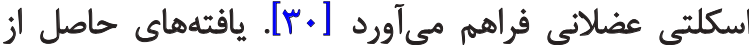

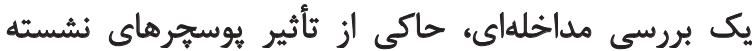

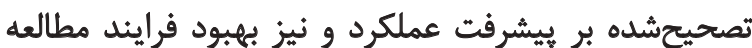

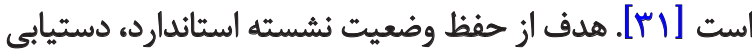

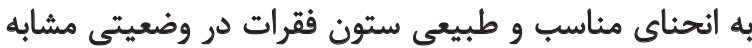

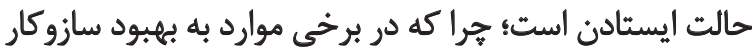

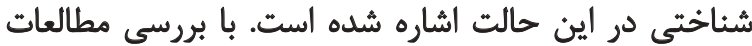

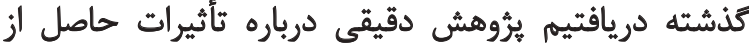

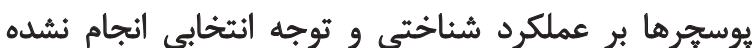

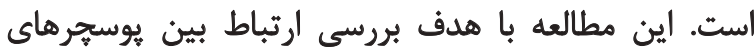

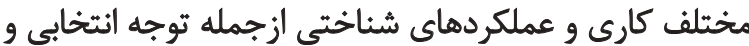

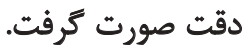

مقلمه

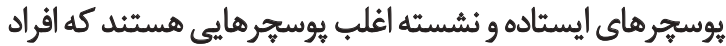

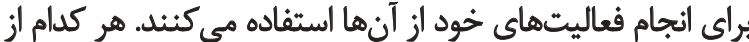

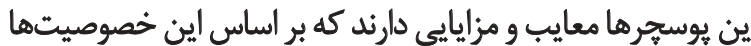

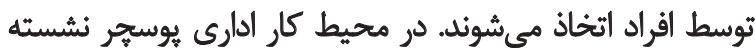

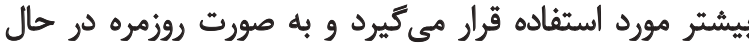

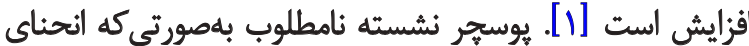

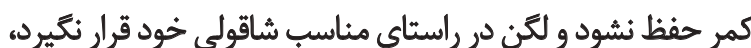

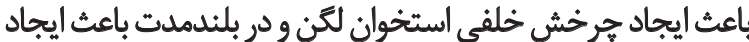

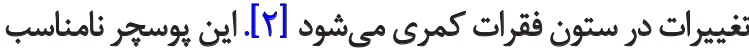

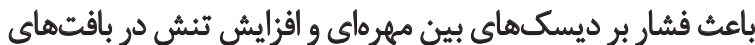

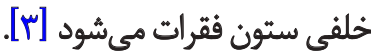

يوسجر مناسب عامل برقرارى تعادل، محافظت از ساختار

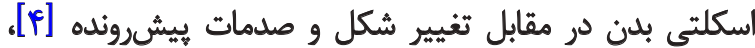

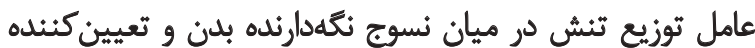

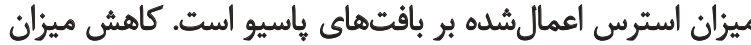

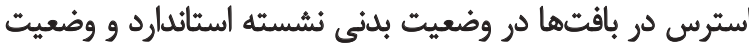

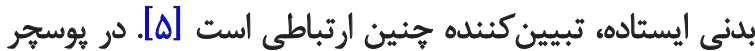

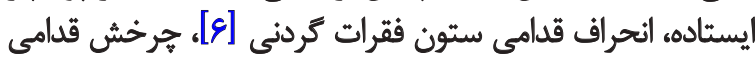

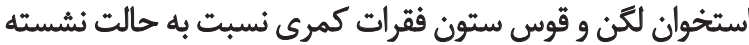

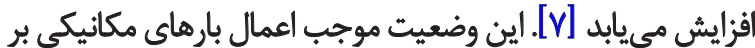

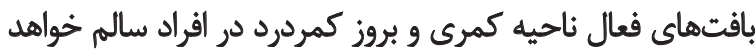

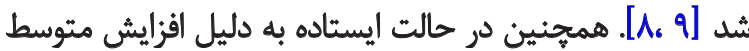

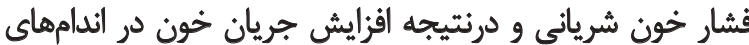

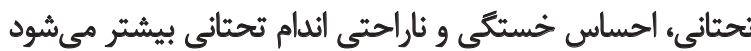

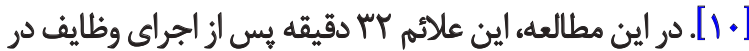

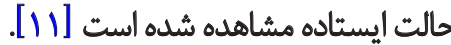

با وجود اينكه يوسجر نامناسب، باعث افزايش بار فيزيكى بر برائر

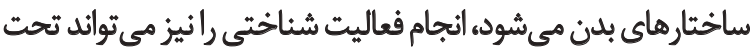

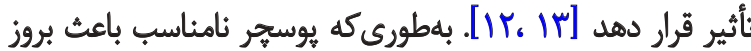

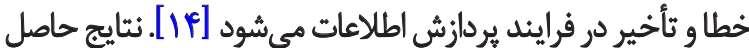

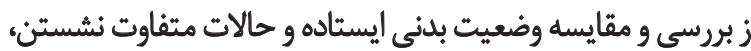

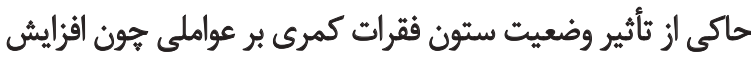

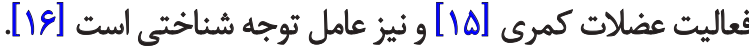

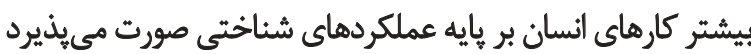

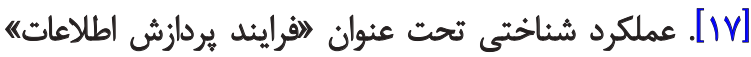

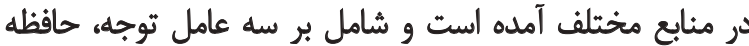

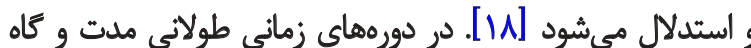

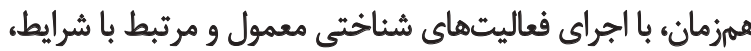

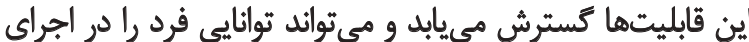

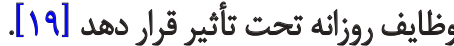
نتايج حاصل از مطالعات، تبيين كنئده ارتباط بين يوسجرهاى 
طي سه مر حله و در سه ايستًاه كارى نُشسيته استاندارد، نشسته

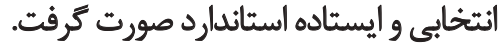

ترتيب قراركرفتئ در ايستكاه كارى به صورت تصادفي صورت

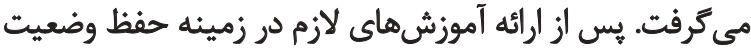

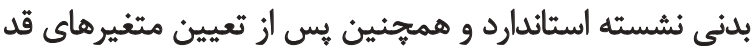

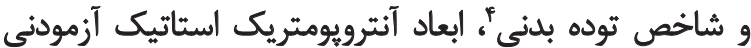

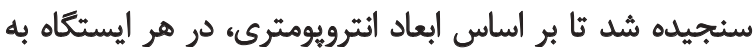

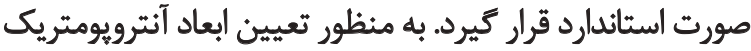

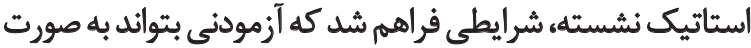

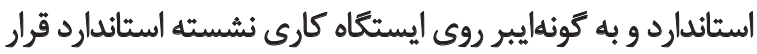

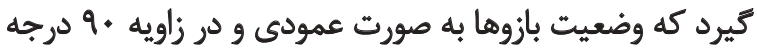

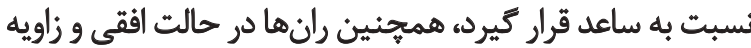

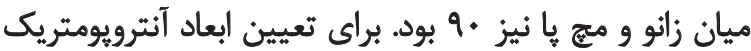

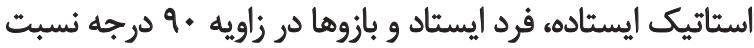

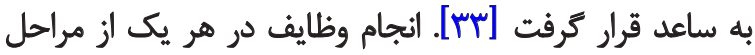

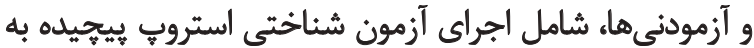

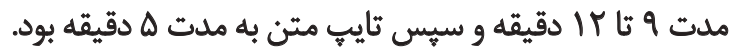

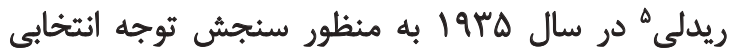

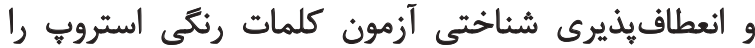

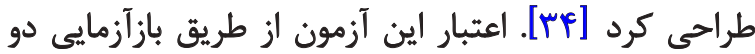

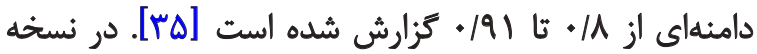

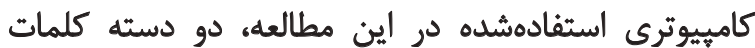

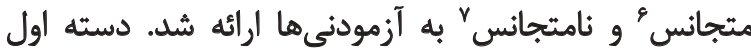

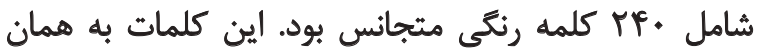

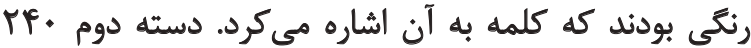

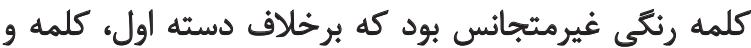

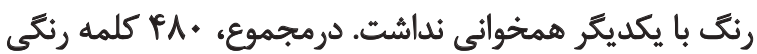

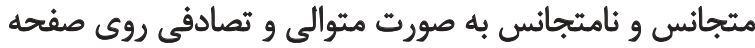

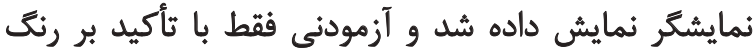

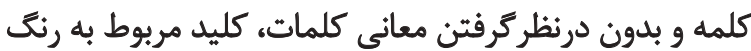
مشخصصشده بر روى صفحه كليد را فشار مي دئداد. مدتزمانى كه يَّ از ارائه محرك (نام كلمه)، كليد مربوطه

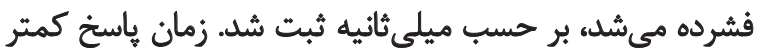

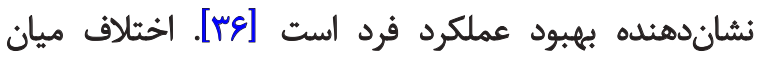

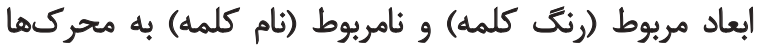

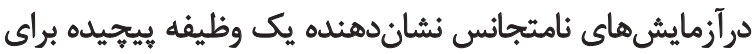

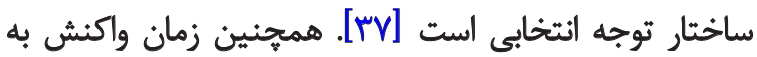

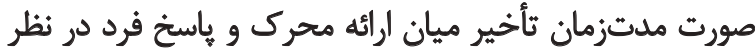

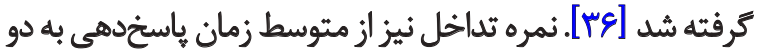

4. Body Mass Index

5. John Ridley Stroop

6. Congruent

7. Incongruent
ووش بورسى

r. مطالعه حاضر از نوع نيمهتجربي است كه روى دانشجويان

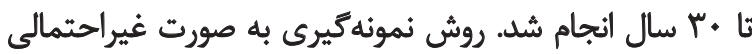

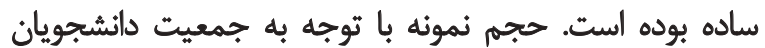

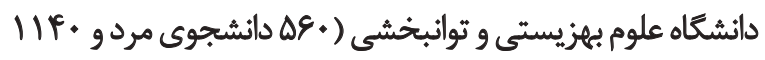

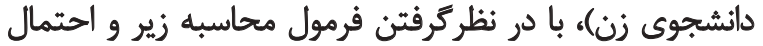

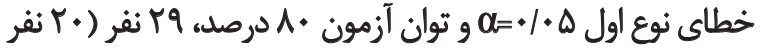

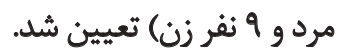
$n=\frac{2 \sigma_{d}^{2}\left(Z_{1-\alpha / 2}+Z_{1-\beta}\right)^{2}}{\delta^{2}}$

معيار ورود به مطالعه شامل مبتّلانبودن به بيمارىهاي قلبي

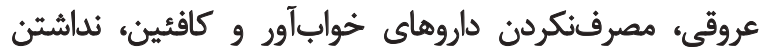
آسيب يا سابقه عمل جراحي در بخشي هاي مختلف سيتون فقرات

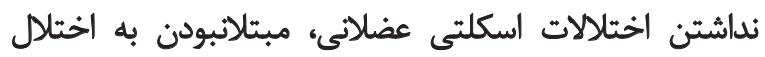
كوررنكى و وجود حساسيت طبيعى نسبت به قابليت تشخيص رنكها، برخوردارى از سرعت تايب متوسط (نداشتن مهارت

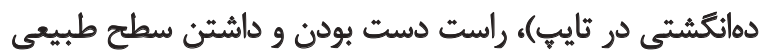

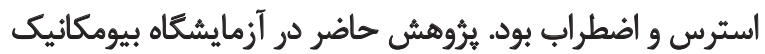

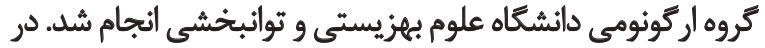

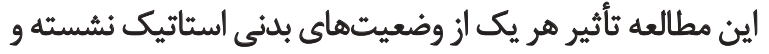

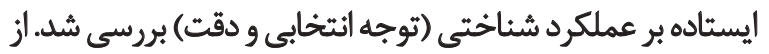

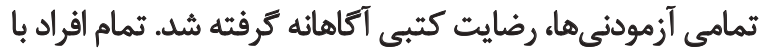

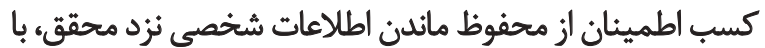

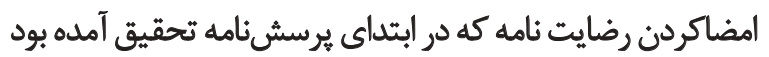

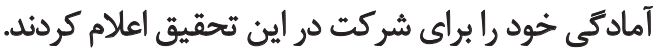
در اين ئروهش سطح اضطراب و استرس شركت كنيدكان به

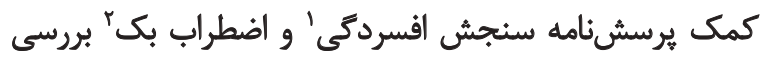

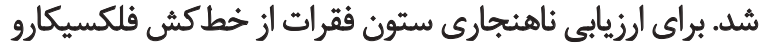

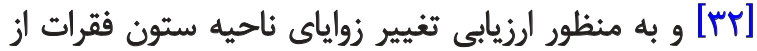

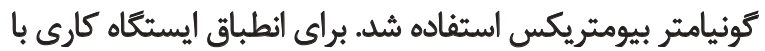

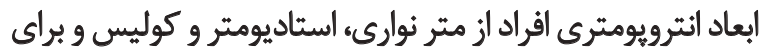

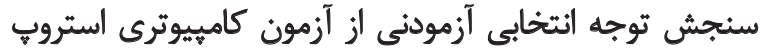

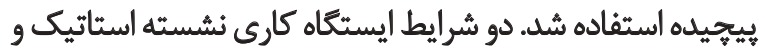

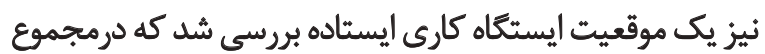

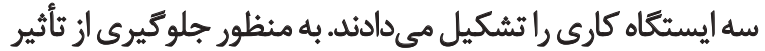

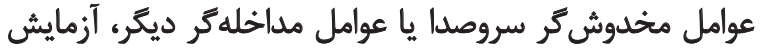

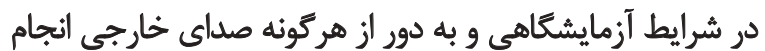

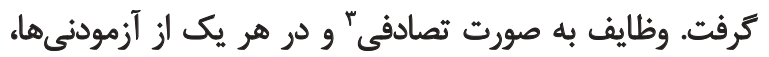

1. Beck Depression Inventory questionnaire

2. Beck Anxiety Inventory questionnaire

3. Random Sampling 


$$
\text { (P=. } 1 \cdot .1)
$$

با مقايسه مقادير مربوط به ميانكين مدتزمان واكنش براي

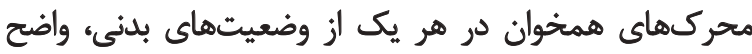

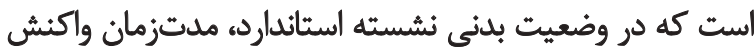

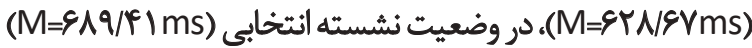

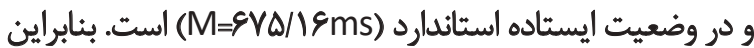

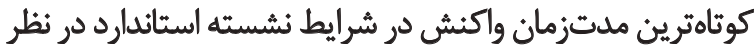

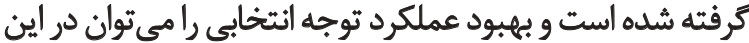

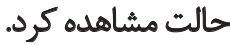

همجئين در ميان ايستأهاي كارى، از نظر مدتزمان

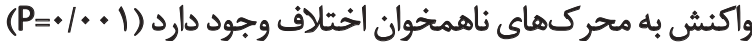

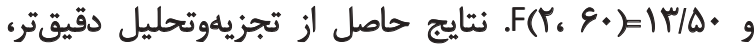

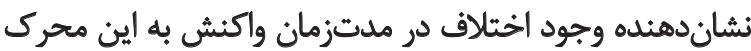

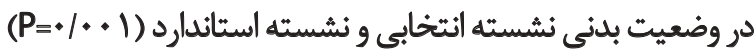

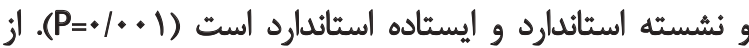

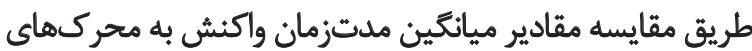

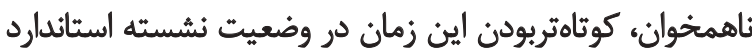

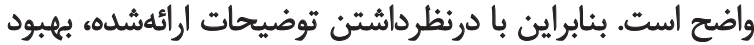

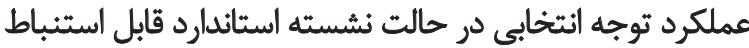

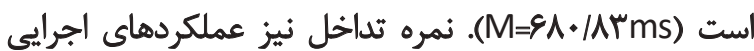

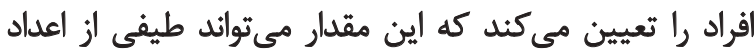

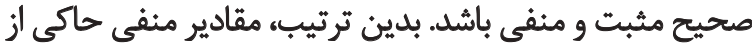

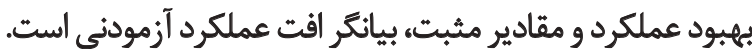
باتوجه به نتايج ارائهده در جدول شماره أ، مى توان دريافت كه

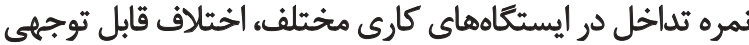

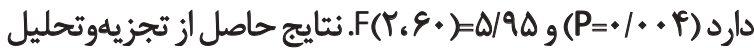

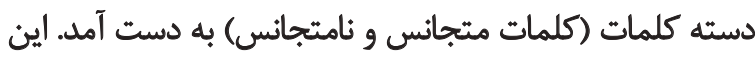

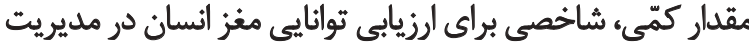

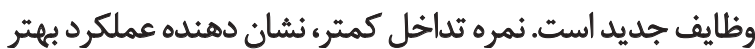

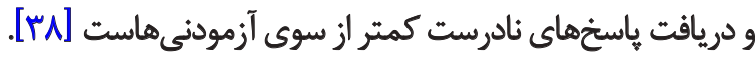
يس از اين آزمون، عملكرد تايّ متن ساده بدين صورت اجرا

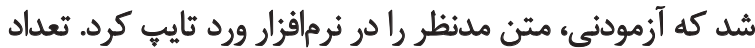

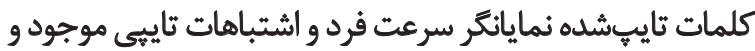

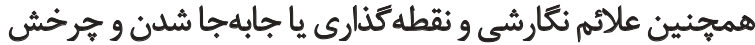

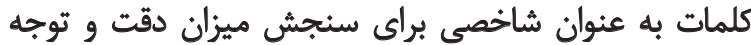

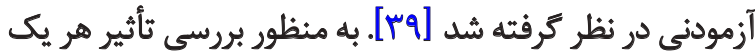

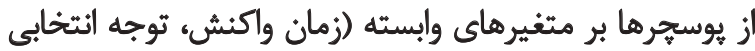

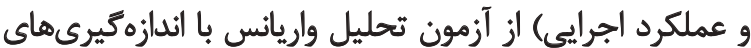

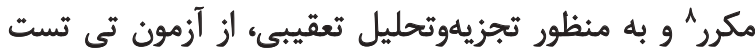

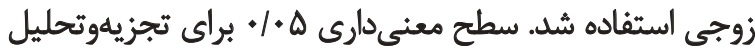

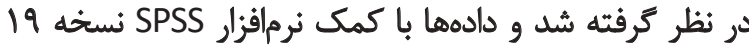
تجزيهوتحليل شد.

يافتهها

نتايج زمان واكنش به محركهاى همخوان نشاندهنده

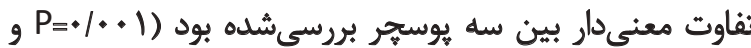

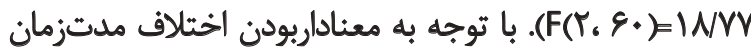

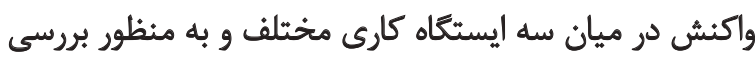

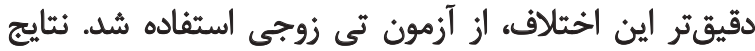

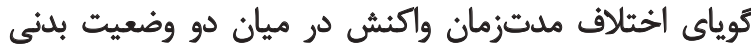

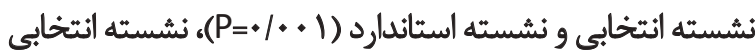

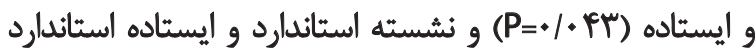

\section{ANOVA with repeated measure}

جدول (. مقايسه يوسجرها بر اساس نثتايج عوامل توجه انتخابى و زمان واكنش

\begin{tabular}{|c|c|c|c|c|c|}
\hline نشسته و ايستاده استاثدارد & نشسته اتثخابي و نشسته استثاثدارو & نشسته و ايستاده أتَّابيى & $\mathbf{F}$ & P* & متغير \\
\hline $.1 .+1$ & $.1 . .1$ & $.1 .4 T$ & $F(E \cdot, Y)=W W$ & $.1 . .1$ & زمان واكثش به محركىهائ \\
\hline $.1 . .1$ & $.1 . .1$ & & $F\left(\varphi_{0}, Y\right)=I T / \Delta$. & .10 .1 & زمان واكثش به محرك كهائ \\
\hline$\leftrightarrow * r$ & $.1 .0 \%$ & & $F(\mathcal{G} \cdot, Y)=\Delta / \neg \Delta$ & $\%$ & نمره تداخل \\
\hline
\end{tabular}

توانبخننى

جدول Y. مقايسه يوسجر هاى مختلف نشسته انتخابي، تُشسته استاندارد و ايسئاده استاندارد بر اساس عملكرد دقت

\begin{tabular}{|c|c|c|c|c|c|}
\hline ايستاده و نشسته استاندارد & نشسته اتثخابى و نشسته استاندارد & نشسته انتخابي و ايستاده & $\mathbf{F}$ & P* & متغير \\
\hline & & & $F(\varepsilon \cdot, Y)=V / \varepsilon q$ &.$/ 194$ & تعلداد كلمات تايتي شده \\
\hline$\% 1$ & $* * 1$ & & $F(\varepsilon \cdot r)=T \& / T$ & $.1 . .1$ & تعداد الشتباهات تاييين" \\
\hline
\end{tabular}

توانبخننى 
و ابزار متفاوت ارزيابي عملكرد افراد از طريق خود اظهارى مي توانواند

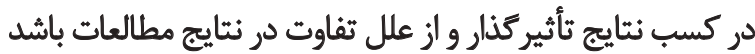

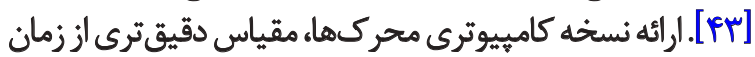

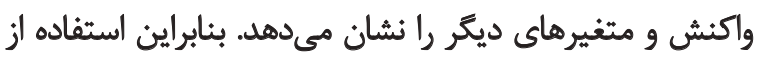

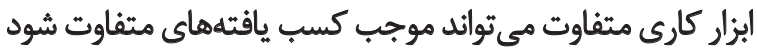

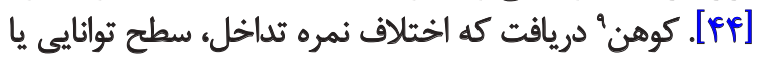

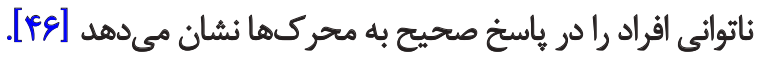

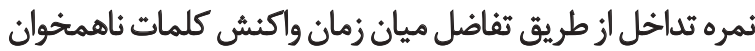

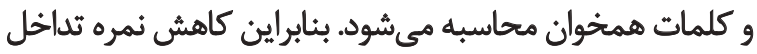

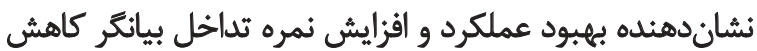

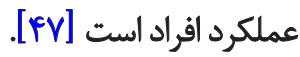

نتايج حاصل از مطالعه حاضر نشان داد ميان يوسجرهاي

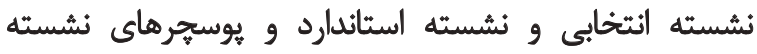

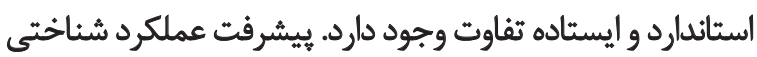

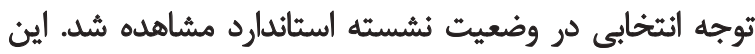

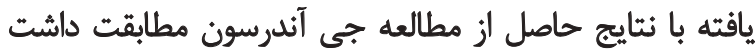

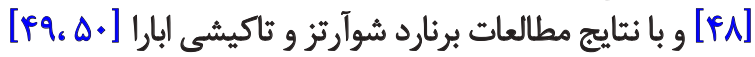

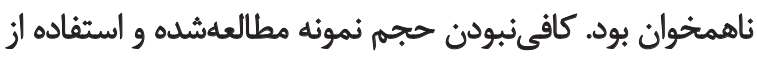

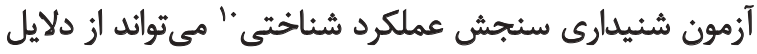

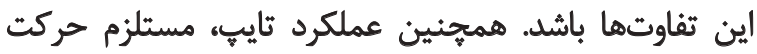

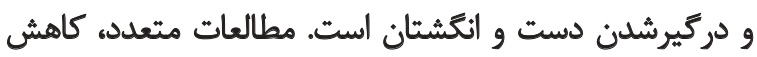

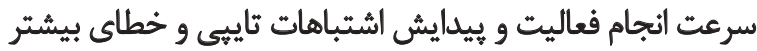

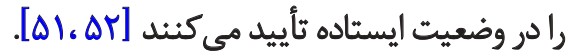

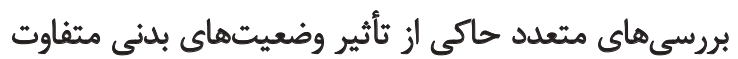

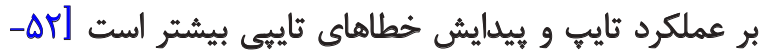
LAf

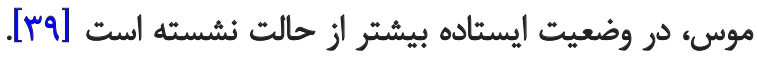

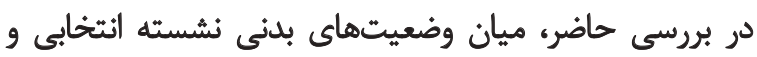

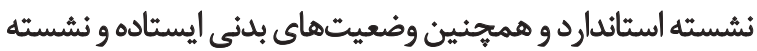

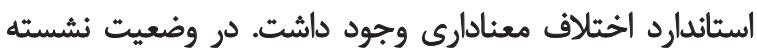

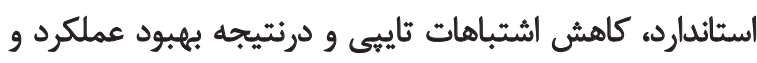
افزايش دقت مشاهده شد.

يافته مذكور با نتايج حاصل از مطالعات ديانا كاميشر و وارن

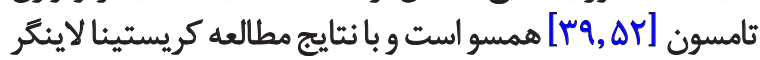

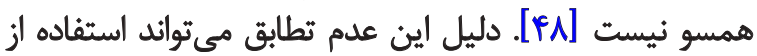

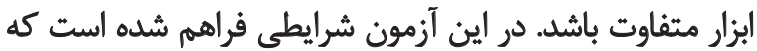

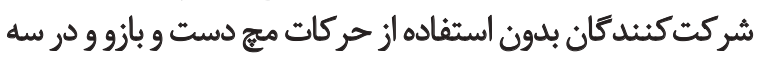

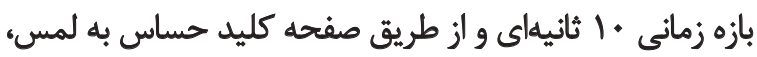

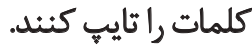

بلهوركلى در افراد مخثلف و با توجه به نتايج حاصل از

9. Cohen

10. Paced Auditory Serial Addition
تعقيبى نشان داد اين تفاوت در ميان وضعيتهاي بدنى (نشسته

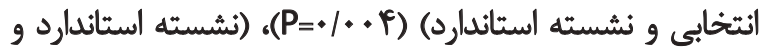

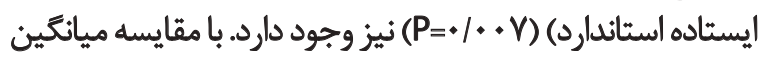

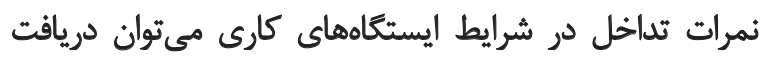

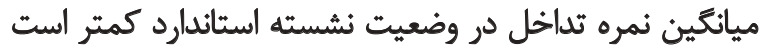

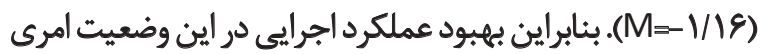

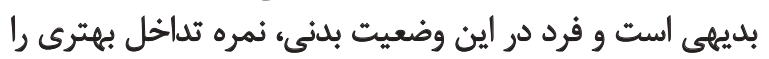
كسب مى كند و درنتيجه عملكرد بهترى دارد. بدري

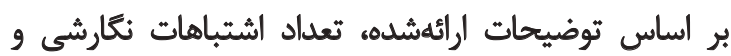

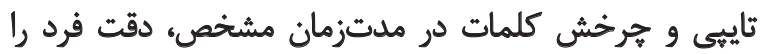

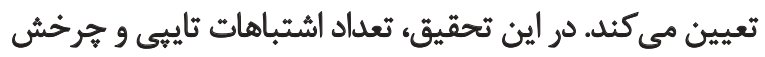

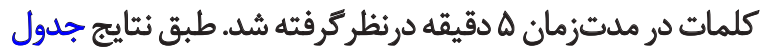

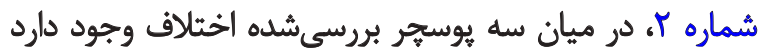

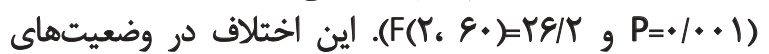

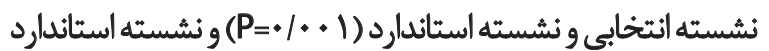

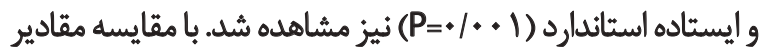

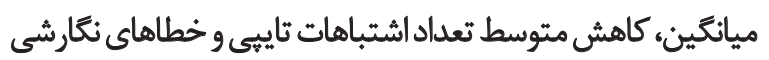

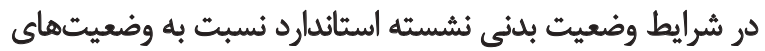

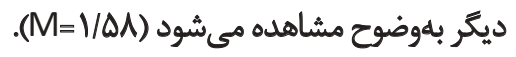

ث̊!

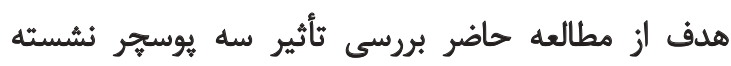

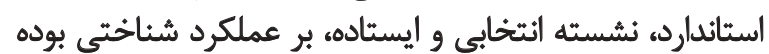

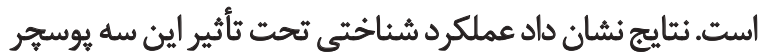

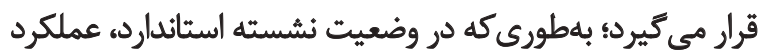

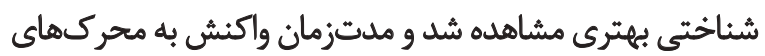

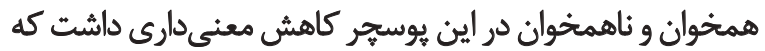

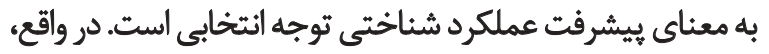

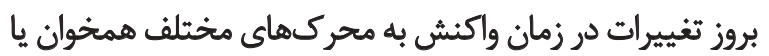

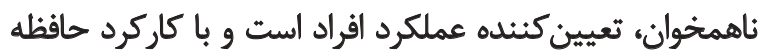

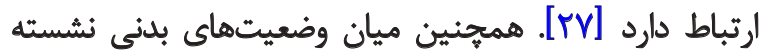

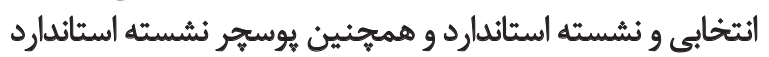

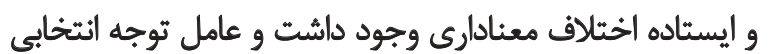

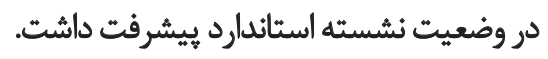

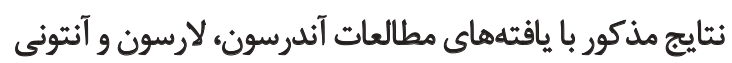

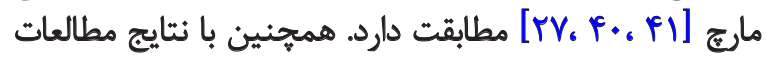

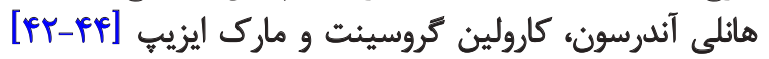

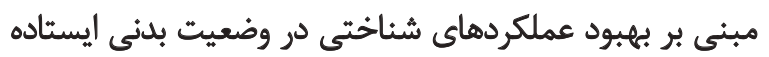

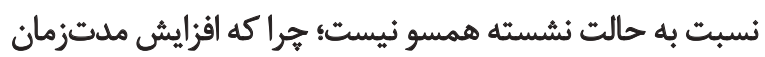

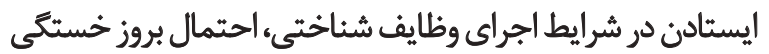

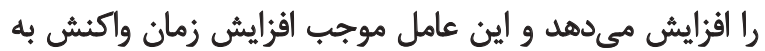

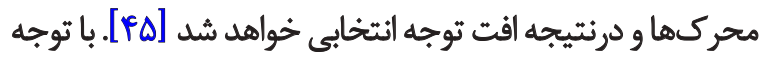

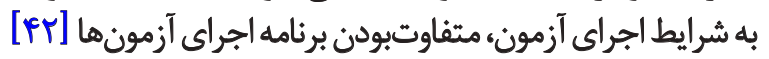




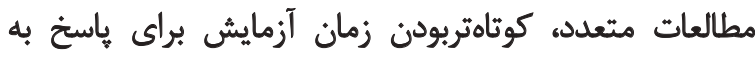

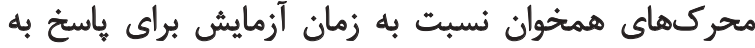

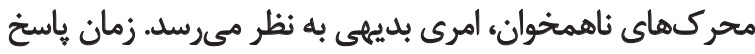

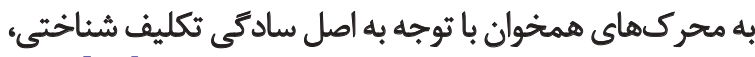

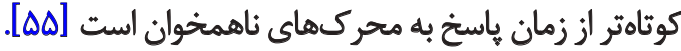
اين نكته با نتايج حاصل از مطالعه حاضر مطابقت دارد. نكته

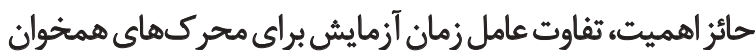

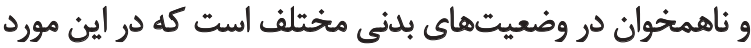

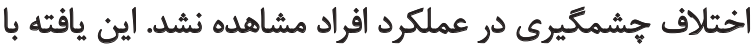

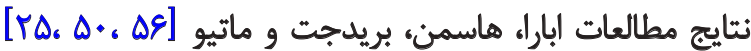

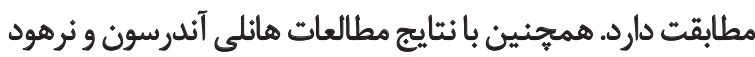

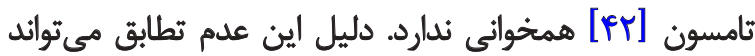

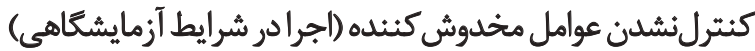

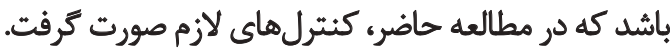

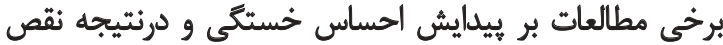

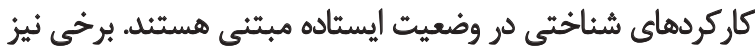

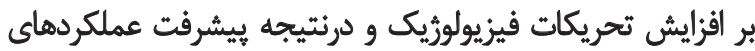

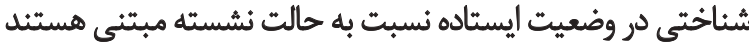

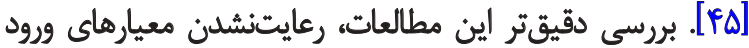

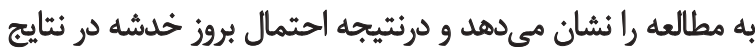

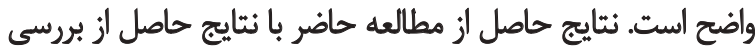

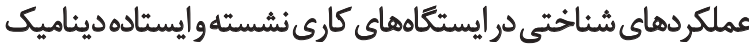

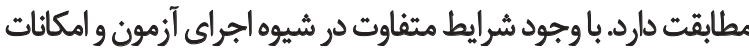

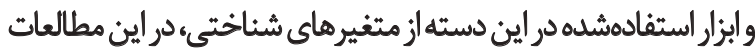
نيز تغيير جشمخيرى مشاهده نشده است [هV]

در زمينه محدوديتهاى مطالعه در اين بررسى، ميتوان

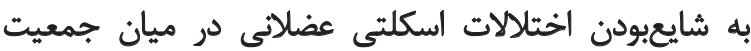

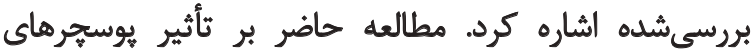

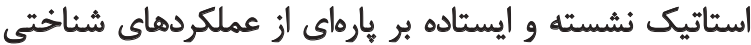

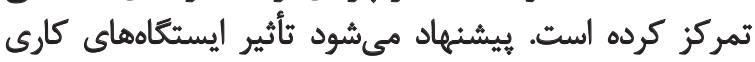

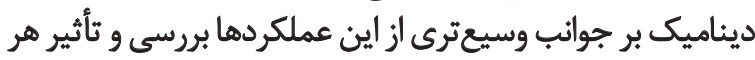

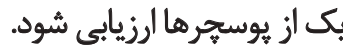

\section{نتيجلكيرى}

نتايج اين مطالعه بيانكر متأثربودن عملكرد شناختى توجه

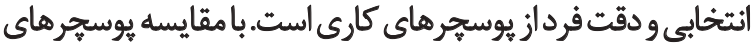
مختلف، عملكرد در وضعيت نشسته استاندارد بهيبود يافت. بارئ

$$
\text { تشكير و قدردافي }
$$

مقاله حاضر از هايانانامه مقطع كارشناسى ارشد خانم شراره

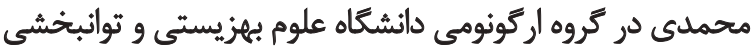
تهران كرفته شده است. 


\section{References}

[1] Yektaee T, Tabatabaee Ghomshe F, Piri L. [The effect of ergonomic principles education on musculoskeletal disorders among computer users (Persian)]. Archives of Rehabilitation. 2013; 13(4):108-16.

[2] Endo K, Suzuki H, Nishimura H, Tanaka H, Shishido T, Yamamoto K. Sagittal lumbar and pelvic alignment in the standing and sitting positions. Journal of Orthopaedic Science. 2012; 17(6):682-6. doi: 10.1007/s00776-012-0281-1

[3] Videman T, Nurminen M, Troup JDG. Lumbar spinal pathology in cadaveric material in relation to history of back pain, occupation, and physical loading. Spine. 1990; 15(8):728-40. doi: 10.1097/00007632-199008000-00002

[4] Malepe MM, Goon DT, Anyanwu FC. The relationship between postural deviations and body mass index among university students. Biomedical Research. 2015; 26(3):437-42.

[5] Castanharo R, Duarte M, McGill S. Corrective sitting strategies: An examination of muscle activity and spine loading. Journal of Electromyography and Kinesiology. 2014; 24(1):114-9. doi: 10.1016/j.jelekin.2013.11.001

[6] Silva AG, Punt TD, Sharples P, Vilas Boas JP, Johnson MI. Head posture and neck pain of chronic nontraumatic origin: A comparison between patients and pain free persons. Archives of Physical Medicine and Rehabilitation. 2009; 90(4):669-74. doi: 10.1016/j. apmr.2008.10.018

[7] Claeys K, Brumagne S, Deklerck J, Vanderhaeghen J, Dankaerts W. Sagittal evaluation of usual standing and sitting spinal posture. Journal of Bodywork and Movement Therapies. 2016; 20(2):32633. doi: 10.1016/j.jbmt.2015.10.002

[8] Marshall PWM, Patel H, Callaghan JP. Gluteus medius strength, endurance, and coactivation in the development of low back pain during prolonged standing. Human Movement Science. 2011; 30(1):63-73. doi: 10.1016/j.humov.2010.08.017

[9] Mo'tamed Zadeh M, Shafiei Motlagh M, Darvishi E. [Ergonomics intervention in unit blast furnace of a typical steel company (Persian)]. Archives of Rehabilitation. 2013; 14(3):80-7.

[10] Murata J, Murata S, Horie J, Ohtao H, Miyazaki J. Relationship between orthostatic blood pressure changes and postural sway when standing up from a chair in older adult females. International Journal of Gerontology. 2012; 6(3):182-6. doi: 10.1016/j. ijge.2012.01.011

[11] Antle DM, Côté JN. Relationships between lower limb and trunk discomfort and vascular, muscular and kinetic outcomes during stationary standing work. Gait \& Posture. 2013; 37(4):615-9. Doi: 10.1016/j.gaitpost.2012.10.004

[12] Novak D, Mihelj M, Munih M. Psychophysiological responses to different levels of cognitive and physical workload in haptic interaction. Robotica. 2010; 29(3):367-74. doi: 10.1017/ s0263574710000184

[13] Sharif Nia SH, Hagh Doust AA, Haji Hosseini F, Hojjati H, Javan Amoli M. [Effect of occupational and psychological factors in back pain nurses in Amol City (Persian)]. Archives of Rehabilitation. 2012; 12(4):93-101.
[14] DiDomenico A, Nussbaum MA. Effects of different physical workload parameters on mental workload and performance. International Journal of Industrial Ergonomics. 2011; 41(3):25560. doi: 10.1016/j.ergon.2011.01.008

[15] Claus AP, Hides JA, Moseley GL, Hodges PW. Different ways to balance the spine. Spine. 2009; 34(6):208-14. doi: 10.1097/ brs.0b013e3181908ead

[16] Lajoie Y, Teasdale N, Bard C, Fleury M. Attentional demands for static and dynamic equilibrium. Experimental Brain Research. 1993; 97 (1). doi: 10.1007/bf00228824

[17] Ando S, Kokubu M, Yamada Y, Kimura M. Does cerebral oxygenation affect cognitive function during exercise. European Journal of Applied Physiology. 2011; 111(9):1973-82. doi: 10.1007/s00421-011-1827-1

[18] Fitzsimmons PT, Maher JP, Doerksen SE, Elavsky S, Rebar AL, Conroy DE. A daily process analysis of physical activity, sedentary behavior, and perceived cognitive abilities. Psychology of Sport and Exercise. 2014; 15(5):498-504. doi: 10.1016/j.psychsport.2014.04.008

[19] Neupert SD, Almeida DM, Mroczek DK, Spiro A. Daily stressors and memory failures in a naturalistic setting: Findings from the va normative aging study. Psychology and Aging. 2006; 21(2):424-9. doi: 10.1037/0882-7974.21.2.424

[20] Goodenough DR, Oltman PK, Sigman E, Cox PW. The rodand frame illusion in erect and supine observers. Perception \& Psychophysics. 1981; 29(4):365-70. doi: 10.3758/bf03207346

[21] Lipnicki DM, Byrne DG. Thinking on your back: Solving anagrams faster when supine than when standing. Cognitive Brain Research. 2005; 24(3):719-22. doi: 10.1016/j.cogbrainres.2005.03.003

[22] Bantoft C, Summers MJ, Tranent PJ, Palmer MA, Cooley PD, Pedersen SJ. Effect of standing or walking at a workstation on cognitive function. Human Factors: The Journal of the $\mathrm{Hu}-$ man Factors and Ergonomics Society. 2015; 58(1):140-9. doi $10.1177 / 0018720815605446$

[23] Colcombe SJ, Erickson KI, Scalf PE, Kim JS, Prakash R, McAuley E, et al. Aerobic exercise training increases brain volume in aging humans. The Journals of Gerontology Series A: Biological Sciences and Medical Sciences. 2006; 61(11):1166-70. doi: 10.1093/gerona/61.11.1166

[24] Barella LA, Etnier JL, Chang YK. The immediate and delayed effects of an acute bout of exercise on cognitive performance of healthy older adults. Journal of Aging and Physical Activity. 2010; 18(1):87-98. doi: 10.1123/japa.18.1.87

[25] Russell BA, Summers MJ, Tranent PJ, Palmer MA, Cooley PD, Pedersen SJ. A randomised control trial of the cognitive effects of working in a seated as opposed to a standing position in office workers. Ergonomics. 2015; 59(6):737-44. doi: 10.1080/00140139.2015.1094579

[26] Naccarato M, Leviner S, Proehl CJ, Cen C, Barnason FS, Brim C, et al.. Clinical practice guideline: Orthostatic vital signs. Illinois: Emergency Nurses Association; 2011. 
[27] MC S. Assessing the effect of self positioning on cognitive executive function. Journal of Ergonomics. 2012; 2(4). doi: 10.4172/2165-7556.1000110

[28] Healy GN, Matthews CE, Dunstan DW, Winkler EAH, Owen $\mathrm{N}$. Sedentary time and cardio-metabolic biomarkers in US adults: NHANES 2003-06. European Heart Journal. 2011; 32(5):590-7. doi: 10.1093/eurheartj/ehq451

[29] Gallagher KM, Campbell T, Callaghan JP. The influence of a seated break on prolonged standing induced low back pain development. Ergonomics. 2014; 57(4):555-62. doi: 10.1080/00140139.2014.893027

[30] Straker L, Mathiassen SE. Increased physical work loads in modern work: A necessity for better health and performance. Ergonomics. 2009; 52(10):1215-25. doi: 10.1080/00140130903039101

[31] Haynes S, Williams K. Impact of seating posture on user comfort and typing performance for people with chronic low back pain. International Journal of Industrial Ergonomics. 2008; 38(1):35-46. doi: 10.1016/j.ergon.2007.08.003

[32] Khalkhali M, Parnianpour M, Karimi H, Mobini B, Kazemnejhad A. The validity and reliability of measurement of thoracic kyphosis using flexible ruler in postural hyper-kyphotic patients. Journal of Biomechanics. 2006; 39:S541. doi: 10.1016/s00219290(06)85226-7

[33] Pheasant S, Haslegrave CM. Bodyspace: Anthropometry, ergonomics and the design of work. Florida: CRC Press; 2016.

[34] Stroop JR. Studies of interference in serial verbal reactions. Journal of Experimental Psychology. 1935; 18(6):643-62. doi: $10.1037 / \mathrm{h} 0054651$

[35] Lezak MD. Neuropsychological assessment. Oxford: Oxford University Press; 2004.

[36] Van Boxtel MPJ, Ten Tusscher MPM, Metsemakers JFM, Willems B, Jolles J. Visual determinants of reduced performance on the stroop color word test in normal aging individuals. Journal of Clinical and Experimental Neuropsychology. 2001; 23(5):620-7. doi: $10.1076 /$ jcen.23.5.620.1245

[37] Joseph JS, Chun MM, Nakayama K. Attentional requirements in a "preattentive" feature search task. Nature. 1997; 387(6635):8057. doi: $10.1038 / 42940$

[38] Sørensen L, Plessen KJ, Adolfsdottir S, Lundervold AJ. The specificity of the Stroop interference score of errors to ADHD in boys. Child Neuropsychology. 2013; 20(6):677-91. doi: 10.1080/09297049.2013.855716

[39] Commissaris DACM, Könemann R, Hiemstra van Mastrigt S, Burford EM, Botter J, Douwes M, et al. Effects of a standing and three dynamic workstations on computer task performance and cognitive function tests. Applied Ergonomics. 2014; 45(6):15708. doi: 10.1016/j.apergo.2014.05.003

[40] Larson MJ, LeCheminant JD, Hill K, Carbine K, Masterson T, Christenson E. Cognitive and typing outcomes measured simultaneously with slow treadmill walking or sitting: Implications for treadmill desks. PLOS ONE. 2015; 10(4):e0121309. doi: 10.1371/ journal.pone. 0121309
[41] Marsh AP, Geel SE. The effect of age on the attentional demands of postural control. Gait \& Posture. 2000; 12(2):105-13. doi: 10.1016/s0966-6362(00)00074-6

[42] Anderson Hanley C, Arciero PJ, Westen SC, Nimon J, Zimmerman E. Neuropsychological benefits of stationary bike exercise and a cybercycle exergame for older adults with diabetes: An exploratory analysis. Journal of Diabetes Science and Technology. 2012; 6(4):849-57. doi: 10.1177/193229681200600416

[43] Grunseit AC, Chau JYY, Van der Ploeg HP, Bauman A. Thinking on your feet: A qualitative evaluation of sit stand desks in an Australian workplace. BMC Public Health. 2013; 13(1). doi: 10.1186/1471-2458-13-365

[44] Isip MI. Effect of a standing body position during college students' exam. Industrial Engineering \& Management Systems. 2014; 13(2):185-92

[45] Caldwell JA, Prazinko B, Caldwell JL. Body posture affects electroencephalographic activity and psychomotor vigilance task performance in sleep deprived subjects. Clinical Neurophysiology. 2003; 114(1):23-31. doi: 10.1016/s1388-2457(02)00283-3

[46] Cohen JD, Barch DM, Carter C, Servan Schreiber D. Contextprocessing deficits in schizophrenia: Converging evidence from three theoretically motivated cognitive tasks. Journal of Abnormal Psychology. 1999; 108(1):120-33. doi: 10.1037/0021$843 x .108 .1 .120$

[47] Egeland J, Langfjæran T. Differentiating malingering from genuine cognitive dysfunction using the trail making test ratio and stroop interference scores. Applied Neuropsychology. 2007; 14(2):113-9. doi: 10.1080/09084280701319953

[48] Andersen KJ. Are you sitting down? Towards cognitive perfor mance informed design. ECS Tech Report. 2012; 340535:1-3.

[49] Schwartz B. Cognitive and biomechanical effects of postural changes in office environments. Karlsruhe: Upper Austria University of Applied Sciences; 2015.

[50] Ebara T, Kubo T, Inoue T, Murasaki GI, Takeyama H, Sato T, et al. Effects of adjustable sit-stand VDT workstations on workers' musculoskeletal discomfort, alertness and performance. Industrial health. 2008; 46(5):497-505. doi: 10.2486/indhealth.46.497

[51] Ohlinger CM, Horn TS, Berg WP, Cox RH. The effect of active workstation use on measures of cognition, attention, and motor skill. Journal of Physical Activity and Health. 2011; 8(1):119-25. doi: 10.1123/jpah.8.1.119

[52] Thompson WG, Levine JA. Productivity of transcriptionists using a treadmill desk. Work. 2011; 40(4):473-7. doi: 10.3233/ WOR-2011-1258

[53] Funk RE, Taylor ML, Creekmur CC, Ohlinger CM, Cox RH, Berg WP. Effect of walking speed on typing performance using an active workstation. Perceptual and Motor Skills. 2012; 115(1):309-18. doi: 10.2466/06.23.26.pms.115.4.309-318

[54] Straker L, Levine J, Campbell A. The effects of walking and cycling computer workstations on keyboard and mouse performance. Human Factors: The Journal of the Human Factors and Ergonomics Society. 2009; 51(6):831-44. doi: $10.1177 / 0018720810362079$ 
[55] Eidels A, Townsend JT, Algom D. Comparing perception of Stroop stimuli in focused versus divided attention paradigms: Evidence for dramatic processing differences. Cognition. 2010; 114(2):129-50. doi: 10.1016/j.cognition.2009.08.008

[56] Husemann B, Von Mach CY, Borsotto D, Zepf KI, Scharnbacher J. Comparisons of musculoskeletal complaints and data entry between a sitting and a sit stand workstation paradigm. Human Factors: The Journal of the Human Factors and Ergonomics Society. 2009; 51(3):310-20. doi: 10.1177/0018720809338173

[57] John D, Bassett D, Thompson D, Fairbrother J, Baldwin D. Effect of using a treadmill workstation on performance of simulated office work tasks. Journal of Physical Activity and Health. 2009; 6(5):617-24. doi: 10.1123/jpah.6.5.617 